\title{
Vector Potential Quantization and the Quantum Vacuum
}

\author{
Constantin Meis \\ National Institute for Nuclear Science and Technology, Centre d'Etudes de Saclay (CEA), 91191 Gif-sur-Yvette, France \\ Correspondence should be addressed to Constantin Meis; constantin.meis@cea.fr
}

Received 17 January 2014; Revised 4 June 2014; Accepted 4 June 2014; Published 19 June 2014

Academic Editor: Ali Hussain Reshak

Copyright (C) 2014 Constantin Meis. This is an open access article distributed under the Creative Commons Attribution License, which permits unrestricted use, distribution, and reproduction in any medium, provided the original work is properly cited.

\begin{abstract}
We investigate the quantization of the vector potential amplitude of the electromagnetic field to a single photon state starting from the fundamental link equations between the classical electromagnetic theory and the quantum mechanical expressions. The resulting wave-particle formalism ensures a coherent transition between the classical electromagnetic wave theory and the quantum representation. A quantization constant of the photon vector potential is defined. A new quantum vacuum description results directly in having very low energy density. The calculated spontaneous emission rate and Lambs shift for the $n S$ states of the hydrogen atom are in agreement with quantum electrodynamics. This low energy quantum vacuum state might be compatible with recent astrophysical observations.
\end{abstract}

\section{Introduction}

It is well known [1-5] that the Hamiltonian radiation in quantum electrodynamics (QED) writes

$$
H=\sum_{k, \lambda} \hbar \omega_{k}\left(\widehat{N}_{k \lambda}+\frac{1}{2}\right),
$$

where $\hbar$ is Planck's reduced constant $(h / 2 \pi)$ and $\widehat{N}_{k \lambda}=a_{k \lambda}^{+} a_{k \lambda}$ is the number of photon operator with $a_{k \lambda}$ and $a_{k \lambda}^{+}$being the annihilation and creation operators of a $k$ mode and $\lambda$ polarization photon, respectively, with angular frequency $\omega_{k}$. In the absence of photons, (1) leads to the QED singularity of infinite vacuum energy [5-7] $\sum_{k, \lambda} \hbar \omega_{k} / 2$. It has been demonstrated that when considering only the QED quantum vacuum, even with "reasonable" frequency cut-offs, the corresponding energy density is many orders of magnitude greater than actually observed $[8,9]$ in universe. This situation has been called the quantum vacuum catastrophe entailing the necessity of new theoretical developments.

Indeed, in the last two decades, various studies, developing different models [10-19], have been carried out in order to interpret the recent astrophysical observations which are in contradiction with the quantum electrodynamics (QED) vacuum representation [5-8]. Those studies have revealed the necessity of developing a different approach for the electromagnetic vacuum representation.
In this paper, we enhance the quantization procedure of the electromagnetic field vector potential [20] down to a single photon level in order to better understand the nature of the photon and that of the vacuum state. The resulting quantum vacuum field is a very low energy density medium that can be compatible with the astronomical observations. It is also applied in this paper for the calculation of the spontaneous emission transition rates as well as the Lamb shift of the $n S$ states in the hydrogen atom showing the consistency and complementarity with QED.

\section{Background Theory of the Vector Potential Amplitude of a Single Photon}

We will not consider, here, for the vacuum energy the singularity $\sum_{k, \lambda} \hbar \omega_{k} / 2$ obtained from (1) in the absence of photons since this term drops when using the "normal ordering" for the photon creation and annihilation operators in the quantization process $[5,6,21]$. Furthermore, it has been demonstrated [22] that the Casimir effect can very well be calculated without referring at all to this term. Hence, we start from the fundamental relations defining in a given volume $V$ the transition between the classical electromagnetic wave 
vector potential amplitude and the corresponding quantized field operators [1-7] used in QED:

$$
\begin{aligned}
& A_{k \lambda} \longrightarrow \sqrt{\frac{\hbar}{2 \varepsilon_{0} \omega_{k} V}} a_{k \lambda}, \\
& A_{k \lambda}^{*} \longrightarrow \sqrt{\frac{\hbar}{2 \varepsilon_{0} \omega_{k} V}} a_{k \lambda}^{+},
\end{aligned}
$$

where $\varepsilon_{0}$ is the vacuum electric permittivity.

It is very important to recall that the above link equations result from the energy density equivalence as expressed by the classical electromagnetic wave theory, based on Maxwell's equations, and the representation of photons in QED. In fact, the energy density of $N$ photons in a given volume $V$ equals the mean energy density over a period $2 \pi / \omega_{k}$ of the classical electromagnetic field of a plane wave with angular frequency $\omega_{k}$ according to the relation [9-12]

$$
\begin{aligned}
\left\langle\frac{1}{2}\left(\varepsilon_{0}\left|\vec{E}_{\omega}(\vec{r}, t)\right|^{2}+\frac{1}{\mu_{0}}\left|\vec{B}_{\omega}(\vec{r}, t)\right|^{2}\right)\right\rangle_{\text {period }} \\
\quad=\left\langle 4 \varepsilon_{0} \omega^{2} A_{0}^{2}(\omega) \sin ^{2}(\vec{k} \cdot \vec{r}-\omega t)\right\rangle_{\text {period }} \\
\quad=2 \varepsilon_{0} \omega^{2} A_{0}^{2}(\omega)=\frac{E(\omega)}{V}=\frac{N \hbar \omega}{V},
\end{aligned}
$$

where $\vec{E}_{\omega}(\vec{r}, t)$ and $\vec{B}_{\omega}(\vec{r}, t)$ are the electric and magnetic fields, respectively, of the plane wave, $A_{0}(\omega)$ is the amplitude of the vector potential, $E(\omega)$ is the photons energy, and $\mu_{0}$ is the vacuum magnetic permeability. It is worth noticing that the volume $V$ is an external parameter and the link relations (2) are obtained for $N=1$, that is, for a single photon state. The fact that the photon vector potential depends on an external arbitrary parameter $V$ is rather puzzling.

Considering that the vector potential generally consists of a superposition of $k$ modes and $\lambda$ polarizations, it writes with the well-known expression

$$
\begin{aligned}
& \vec{A}(\vec{r}, t) \\
& =\sum_{k, \lambda} \sqrt{\frac{\hbar}{2 \varepsilon_{0} \omega_{k} V}}\left[a_{k \lambda} \widehat{\varepsilon}_{k \lambda} e^{i\left(\vec{k} \cdot \vec{r}-\omega_{k} t+\theta\right)}+a_{k \lambda}^{+} \widehat{\varepsilon}_{k \lambda}^{*} e^{-i\left(\vec{k} \cdot \vec{r}-\omega_{k} t+\theta\right)}\right],
\end{aligned}
$$

where $\widehat{\varepsilon}_{k \lambda}$ is a complex unit vector of polarisation and $\theta$ is a phase parameter.

Recent experiments $[23,24]$ demonstrated the individuality and indivisibility of photons.

However, it is obvious that the link equations used above for the vector potential amplitude do not result in a welldefined description of individual photons but they are rather valid for a large number of photons in a big volume $V$. This is precisely the reason why in QED the discrete summation over the modes is often replaced by a continuous summation in the wave vector space

$$
\sum_{k} \longrightarrow \frac{V}{8 \pi^{3}} \int 4 \pi k^{2} d k=\frac{V}{2 \pi^{2} c^{3}} \int \omega^{2} d \omega
$$

eliminating by this way the parameter $V$ in all calculations involving the square of the vector potential. The expression (5) is valid whatever the shape of the volume $V$ is, provided that the dimensions of the last one are much bigger than the electromagnetic field wavelengths $[2,5,25]$.

Let us now have a close look at the vector potential amplitude of a single photon. Therein, we recall that a dimensional analysis of the general expression of the vector potential amplitude of an electromagnetic field shows that it should be proportional to the angular frequency $\omega_{k}$. Notice that this is not a hypothesis but a mathematical deduction from Maxwell's equations.

Consequently, for the vector potential of a $k$ mode photon, we should have [20]

$$
\alpha_{0 k}\left(\omega_{k}\right) \propto\left(\text { Constant } \times \omega_{k}\right) \longrightarrow \xi \omega_{k} .
$$

So, the expression of the photon vector potential may now be written in a general plane wave expression

$$
\vec{\alpha}_{\omega_{k}}(\vec{r}, t)=\xi \omega_{k}\left[\widehat{\varepsilon} e^{i\left(\vec{k} \cdot \vec{r}-\omega_{k} t+\varphi\right)}+\widehat{\varepsilon}^{*} e^{-i\left(\vec{k} \cdot \vec{r}-\omega_{k} t+\varphi\right)}\right]
$$

whose propagation occurs within a period $T$ over a wavelength $\lambda$ and repeated successively along the propagation axis. It has been demonstrated [20] that $\alpha_{\omega_{k}}(\vec{r}, t)$ satisfies the wave propagation equation

$$
\vec{\nabla}^{2} \vec{\alpha}_{\omega_{k}}(\vec{r}, t)-\frac{1}{c^{2}} \frac{\partial^{2}}{\partial t^{2}} \vec{\alpha}_{\omega_{k}}(\vec{r}, t)=0,
$$

where $c$ is the velocity of light in vacuum, as well as a linear time differential equation

$$
i \xi \frac{\partial}{\partial t} \vec{\alpha}_{\omega_{k}}(\vec{r}, t)=\widetilde{\alpha}_{0} \vec{\alpha}_{\omega_{k}}(\vec{r}, t),
$$

where the amplitude operator writes $\widetilde{\alpha}_{0}=-i \xi c \vec{\nabla}$.

The mathematical correspondence between the pairs $\{E(\omega), \hbar\} \leftrightarrow\left\{\alpha_{0}(\omega), \xi\right\}$ for a single photon is quite obvious.

Following the expression $(7), \alpha_{\omega_{k}}(\vec{r}, t)$ writes with a waveparticle formalism

$$
\begin{aligned}
\vec{\alpha}_{\omega_{k \lambda}}(\vec{r}, t) & =\omega_{k}\left[\xi \widehat{\varepsilon}_{k \lambda} e^{i\left(\vec{k} \cdot \vec{r}-\omega_{k} t+\varphi\right)}+\xi^{*} \widehat{\varepsilon}_{k \lambda}^{*} e^{-i\left(\vec{k} \cdot \vec{r}-\omega_{k} t+\varphi\right)}\right] \\
& =\omega_{k} \vec{\Xi}_{k \lambda}\left(\omega_{k}, \vec{r}, t\right) \\
\widetilde{\alpha}_{k \lambda} & =\omega_{k}\left[\xi a_{k \lambda} \widehat{\varepsilon}_{k \lambda} e^{i\left(\vec{k} \cdot \vec{r}-\omega_{k} t+\varphi\right)}+\xi^{*} a_{k \lambda}^{+} \widehat{\varepsilon}_{k \lambda}^{*} e^{-i\left(\vec{k} \cdot \vec{r}-\omega_{k} t+\varphi\right)}\right] \\
& =\omega_{k} \widetilde{\Xi}_{k \lambda}\left(\omega_{k}, a_{k \lambda}, a_{k \lambda}^{+}\right),
\end{aligned}
$$

where, for the quantum mechanical formalism, we have used the creation and annihilation operators $a_{k \lambda}^{+}$and $a_{k \lambda}$, respectively, for a $k$ mode and $\lambda$ polarization photon.

The mathematical representation of the wave and particle expressions of the vector potential amplitude operator writes [20]

$$
\begin{aligned}
& \text { wave: }\left\{\widetilde{\alpha}_{0}=-i \xi c \vec{\nabla} ; \widetilde{\alpha}_{0}^{*}=i \xi^{*} c \vec{\nabla}\right\}, \\
& \text { particle: }\left\{\widetilde{\alpha}_{0_{k \lambda}}=\xi \omega_{k} a_{k \lambda} ; \widetilde{\alpha}_{0_{k \lambda}}^{*}=\xi^{*} \omega_{k} a_{k \lambda}^{+}\right\} .
\end{aligned}
$$


An approximate value of the quantization constant $\xi$ has been obtained previously [26]. Consider

$$
|\xi| \propto \frac{\hbar}{4 \pi e c}=1.74710^{-25} \text { Volt m}^{-1} \mathrm{~s}^{2}
$$

where $e$ is the electron charge. Appropriate experiments could attribute a more precise value to $\xi$.

\section{The $\widetilde{\Xi}_{0_{k \lambda}}$ Quantum Vacuum Field Effects}

According to the relations in (10), for $\omega_{k}=0$, the vector potential as well as the energy of a $k$ mode photon vanishes. However, the field $\Xi_{k \lambda}$ does not vanish but is reduced to the vacuum field represented, here, as a quantum mechanical operator:

$$
\widetilde{\Xi}_{0_{k \lambda}}=\xi a_{k \lambda} \widehat{\varepsilon}_{k \lambda} e^{i \varphi}+\xi^{*} a_{k \lambda}^{+} \widehat{\varepsilon}_{k \lambda}^{*} e^{-i \varphi} .
$$

Thus, $\widetilde{\Xi}_{0_{k \lambda}}$ is a real entity of the vacuum state having the dimensions Volt $\mathrm{m}^{-1} \mathrm{~s}^{2}$ corresponding to a vector potential per angular frequency and implying an electric nature of the quantum vacuum. Obviously, $\widetilde{\Xi}_{0_{k \lambda}}$ is a dynamic entity capable of inducing electronic transitions in matter since it is described by a function of $a_{k \lambda}^{+}$and $a_{k \lambda}$ operators.

3.1. Spontaneous Emission. In a rigorous calculation, the Hamiltonian interaction between an atomic electron with dipole moment $\vec{d}=e \vec{r}$ and the vacuum writes $H_{\text {int }}=-\vec{d}$. $(i / \hbar)\left[\vec{A}(\vec{r}, t), \sum_{k, \lambda}(1 / 2) \hbar \omega_{k}\right]$, which vanishes as in the semiclassical description. The physical reason is that, in QED, the vacuum state is not described by a function of $a_{k \lambda}$ and $a_{k \lambda}^{+}$ operators and, consequently, a Hamiltonian interaction for the spontaneous emission cannot be defined. Consequently, as it is well known, the spontaneous emission in QED results from the noncommutation between the $a_{k \lambda}$ and $a_{k \lambda}^{+}$operators rather than from a direct interaction between a bounded electron and the energy of the zero level photons $\sum_{k, \lambda} \hbar \omega_{k} / 2$.

Conversely, for the description of the electron-vacuum interaction, a "vacuum action" operator corresponding to a Hamiltonian interaction per angular frequency between the vacuum field $\widetilde{\Xi}_{0_{k \lambda}}$ and an atomic electron of mass $m_{e}$ and charge $e$ can now be defined as follows:

$$
H_{\omega_{k}}=-i \hbar \frac{e}{m_{e}} \widetilde{\Xi}_{0_{k \lambda}} \cdot \vec{\nabla}
$$

Thus, the amplitude of the transition probability per angular frequency between an initial state $\left|\Psi_{i}, 0\right\rangle$ with total energy $E_{i}=\hbar \omega_{i}$, corresponding to an atom in the state $\left|\Psi_{i}\right\rangle$ with energy $E_{i}$ in vacuum $\left|\ldots, 0_{k \lambda}, \ldots, 0_{k^{\prime}} \lambda^{\prime}, \ldots\right\rangle$, and a final state $\left|\Psi_{f}, n_{k \lambda}\right\rangle$ with total energy $E_{f, n_{k \lambda}}=\hbar \omega_{f}+n_{k \lambda} \hbar \omega_{k}$, representing the atom in the state $\left|\Psi_{f}\right\rangle$ with energy $E_{f}=\hbar \omega_{f}$ in the presence of $n_{k \lambda}$ photons, can be expressed in first order time dependent perturbation theory using the well-known formalism $[1,3,5]$ :

$$
\begin{aligned}
c_{\omega_{k}}^{(1)}(t)=-\frac{e}{m_{e}} \int_{0}^{t}[ & \left\langle\Psi_{f}, n_{k \lambda}\left|\xi \vec{\varepsilon}_{k \lambda} \cdot \vec{\nabla} a_{k \lambda}\right| \Psi_{i}, 0\right\rangle \\
& \times e^{i\left(\omega_{i}-\omega_{f}-n_{k \lambda} \omega_{k}\right) t^{\prime}} \\
& +\left\langle\Psi_{f}, n_{k \lambda}\left|\xi^{*} \vec{\varepsilon}_{k \lambda}^{*} \cdot \vec{\nabla} a_{k \lambda}^{+}\right| \Psi_{i}, 0\right\rangle \\
& \left.\times e^{-i\left(\omega_{i}-\omega_{f}-n_{k \lambda} \omega_{k}\right) t^{\prime}}\right] d t^{\prime} .
\end{aligned}
$$

Since $a_{k \lambda}\left|\Psi_{i}, 0\right\rangle=0$, using Heisenberg's equation of motion $[5,21]$, the scalar product of the last equation corresponding to the creation operator $a_{k \lambda}^{+}$writes

$$
\left\langle\Psi_{f}, n_{k \lambda}\left|\xi^{*} \vec{\varepsilon}_{k \lambda}^{*} \cdot \vec{\nabla} a_{k \lambda}^{+}\right| \Psi_{i}, 0\right\rangle=-\xi^{*} \delta_{1, n_{k \lambda}} m_{e} \omega_{i f} \vec{\varepsilon}_{k \lambda}^{*} \cdot \frac{\vec{r}_{i f}}{\hbar}
$$

with $\vec{r}_{i f}=\left\langle\Psi_{f}|\vec{r}| \Psi_{i}\right\rangle, \omega_{i f}=\omega_{i}-\omega_{f}$, and $\delta_{1, n_{k \lambda}}$ being the Kronecker's delta symbol.

Considering the expression $\xi \propto(\hbar / 4 \pi e c)$ from (12), one gets the spontaneous emission rate in the elementary solid angle $d \Omega$. Consider

$$
W_{i f}=\frac{1}{3 \hbar c^{3}} \frac{e^{2}}{4 \pi \varepsilon_{0}} \omega_{i f}^{3}\left|\vec{r}_{i f}\right|^{2} d \Omega .
$$

This result is in full agreement with previous QED calculations $[1,3,21]$ and shows that the spontaneous emission is mainly due to the creation operator $a_{k \lambda}^{+}$, which, here, is involved in the quantum vacuum expression. Consequently, $\widetilde{\Xi}_{0_{k \lambda}}$ is the skeleton of every photon. According to the relations in (10), a photon appears to be a vacuum perturbation generated when the last one interacts with matter conferring an angular frequency $\omega_{k}$ to $\widetilde{\Xi}_{0_{k \lambda}}$.

3.2. Lamb Shift of the $n S$ Levels of the Atomic Hydrogen. Various calculations of the energy shift of the $n S$ levels of the hydrogen atom have been carried out, each one by considering the vacuum effect on the atomic orbitals through different aspects, mass renormalization [27], vacuum electric field perturbation [28], Feynman's gas interpretation [29], Stark shift [5], and so forth. Here, we give a complementary approach based on the $\widetilde{\Xi}_{0_{k \lambda}}$ vacuum field representation.

In a first approximation, the energy level displacements of the electron bounded states can be seen as a topological effect of the vacuum radiation pressure exerted upon the electronic orbitals. In fact, the motion of a bounded state electron with charge $e$, whose energy is $E_{n l j}$, in the vacuum field $\widetilde{\Xi}_{0_{k \lambda}}$ can be characterised by Bohr's angular frequency:

$$
\omega_{n l j}=\frac{E_{n l j}}{\hbar}=\frac{R_{\infty}}{n^{2} \hbar},
$$

where $n, l$, and $j$ are the quantum numbers of the corresponding orbital entailing the rise in the electron frame of a vector potential amplitude

$$
\alpha_{0(n l j)}=\xi \omega_{(n l j)}
$$


corresponding to "vacuum photons" with energy

$$
4 \pi e c \xi \omega_{n l j}=\hbar \omega_{n l j}
$$

Hence, due to its periodic motion in the field $\widetilde{\Xi}_{0_{k \lambda}}$, an electron experiences in its frame vacuum photons whose pressure per unit surface writes [25]

$$
d P(\mathrm{vac})=\sum_{\lambda} \varepsilon_{0}\left|E_{0}^{(R, L)}\right|^{2} d \Omega
$$

where $\left|E_{0}^{(R, L)}\right|$ is the electric field amplitude of the left $(L)$ and right hand $(R)$ polarized vacuum photons seen by the electron.

From classical electrodynamics, we have $[1,25]$

$$
\begin{aligned}
\left|E_{0}^{(R)}\right|^{2} & =\omega_{n l j}^{2}\left(\left|A_{x_{0}}^{(R)}\right|^{2}+\left|A_{y_{0}}^{(R)}\right|^{2}\right) \\
& =\omega_{n l j}^{2}\left(\left|A_{x_{0}}^{(L)}\right|^{2}+\left|A_{y_{0}}^{(L)}\right|^{2}\right)=\omega_{n l j}^{2}\left(2 \xi^{2} \omega_{n l j}^{2}\right) .
\end{aligned}
$$

The summation over the two circular polarizations and over the whole solid angle $4 \pi$ gives the total vacuum radiation pressure

$$
P(\mathrm{vac})=4 \pi \varepsilon_{0}\left(4 \xi^{2} \omega_{n l j}^{4}\right) \approx \frac{\hbar \omega_{n l j}^{4}}{4 \alpha \pi^{2} c^{3}} .
$$

Hence, if the electron subsists in the effective volume $V_{n l j}$ (eff) of the $n l j$ orbital, the corresponding energy shift due to the vacuum radiation pressure writes

$$
\delta E=P(\mathrm{vac}) V_{n l j}(\mathrm{eff}) \approx \frac{\hbar \omega_{n l j}^{4}}{4 \alpha \pi^{2} c^{3}} V_{n l j}(\mathrm{eff}) .
$$

A delicate operation consists of defining $V_{(n l j)}(\mathrm{eff})$. The $n S$ electronic orbitals, for example, are characterised by a density probability distribution decreasing smoothly with an exponential expression. Furthermore, for the excited electronic $n S$ states, the radial wave functions contain negative values and the electron probability density distribution involves a significant space area with zero values. Hence, in the case of atomic hydrogen for the spherically symmetrical electronic orbitals $n S$, in order not to take into account the space regions where the probability density is zero, the effective volume may be written in a first approximation:

$$
V_{(n S)}(\mathrm{eff}) \approx \frac{1}{n} \frac{4}{3} \pi\left|\left\langle\psi_{n s}|r| \psi_{n s}\right\rangle\right|^{3}
$$

and, following the last two equations, the corresponding frequency of the energy shift

$$
v_{n S}=\frac{\delta E_{(n S)}}{h} \approx \frac{\omega_{n S}^{4}}{6 \alpha \pi^{2} c^{3}} \frac{1}{n}\left|\left\langle\psi_{n s}|r| \psi_{n s}\right\rangle\right|^{3} .
$$

Putting $\omega_{n S}=R_{\infty} / n^{2} \hbar$ with $R_{\infty}=13.606 \mathrm{eV}$ and considering the cube of the mean value of the distance in the hydrogen electronic orbitals $n S^{1}$,

$$
|\langle r\rangle|_{n, l=0}^{3}=\left|\int \Psi_{(n, l=0)} r \Psi_{(n, l=0)}^{*} d^{3} r\right|^{3}=a_{0}^{3}\left(\frac{3 n^{2}}{2}\right)^{3}
$$

with $a_{0}=0.5310^{-10} \mathrm{~m}$ being the first Bohr radius; the frequency of the energy shift writes

$$
v_{n S} \approx \frac{1}{n^{3}} \frac{9 R_{\infty}^{4} a_{0}^{3}}{16 \alpha \pi^{2} c^{3} \hbar^{4}} .
$$

We obtain the Lamb shifts frequencies for the atomic hydrogen $n S$ levels:

$$
\begin{aligned}
& 1 S: \sim 7.96 \mathrm{GHz}(8.2), \quad 2 S: \sim 1000 \mathrm{MHz}(1040), \\
& 3 S: \sim 297 \mathrm{MHz}(303), \quad 4 S: \sim 124 \mathrm{MHz}
\end{aligned}
$$

with the experimental values given in parenthesis. The agreement with the experiment is quite satisfactory for such a simple calculation.

\subsection{Astrophysical Observations and the Quantum Vacuum} Catastrophe. It has been pointed out that recent astronomical observations revealed the vacuum energy density in the universe to be many orders of magnitude $[5,8]$ lower than that predicted by QED. In fact, the studies on the dark energy and the arising constraints have stimulated the creation of new models to remedy the theoretical cosmology difficulties. The modelling of the dark energy as a fluid has led to the classical phantom description [10] which was revealed to be instable from both quantum mechanical and gravitational points of view. Some proposals, among many others, based on phantom cosmology have introduced more consistent dark energy models by introducing quantum effects in general relativity [11], nonlinear gravity-matter interaction [12], revised gravity [13], and effective phantom phase and holography [14]. On the other hand, various studies [15-17] on the contribution of the quantum vacuum state to the dark energy have been carried out based on both QCD (quantum chromodynamics) and QED (quantum electrodynamics) descriptions. However, it has been pointed out by many authors that our understanding of the QCD vacuum state still remains very elusive. Conversely, QED vacuum offers a more comprehensive framework and there is a general scientific effort to analyse the contribution of the electromagnetic field lower energy level to the dark energy.

For a finite universe, which we may consider behaving as a cosmic cavity, $\vec{\Xi}_{k \lambda}\left(\omega_{k}, \vec{r}, t\right)$ gets very small but not zero. In fact, for $\omega_{k}=\left(2 \pi c / \lambda_{k}\right) \rightarrow 0$, the wavelength $\lambda_{k}$ of a $k$ mode photon tends to cosmic dimensions while the energy and the vector potential tend to zero. Thus, we may assume that the universe is filled by a superposition of standing waves or photons with cosmic dimensions corresponding a very low energy density. Under these conditions, considering the cosmological estimations on the extend of the universe, an extremely high number of cosmic standing waves, roughly of the order of $10^{100}$, could correspond to the measured background vacuum energy density which is close to $10^{-9} \mathrm{~J} / \mathrm{m}^{3}$.

\section{Conclusions}

The difficulty raised in QED by the infinite energy of the zero point energy can be lifted by considering the quantization of 
the electromagnetic field vector potential to a single photon $\alpha_{0_{k}}\left(\omega_{k}\right)=\xi \omega_{k}$. This fact is not a model but results directly from Maxwell's equations. The issued theoretical development is in agreement with the experimental evidence according to which the photon is revealed to be an integral and indivisible particle with simultaneous wave-particle properties. We have thus obtained both wave and particle representations of the vector potential whose amplitude is proportional to a constant $\xi$. This entails that the quantum vacuum may be expressed by $\widetilde{\Xi}_{0_{k \lambda}}=\xi a_{k \lambda} \widehat{\varepsilon}_{k \lambda} e^{i \varphi}+\xi^{*} a_{k \lambda}^{+} \widehat{\varepsilon}_{k \lambda}^{*} e^{-i \varphi}$, a dynamic entity capable of inducing electronic transitions in atoms and molecules. We have shown that the spontaneous emission rate and the Lamb shift of the atomic hydrogen $n S$ orbitals can equally be calculated using $\widetilde{\Xi}_{0_{k \lambda}}$. Furthermore, for a finite universe, cosmic standing waves composed of $\widetilde{\Xi}_{0}$ could give rise to a very low energy density, compatible with recent vacuum measurements. This opens perspectives for new experiments in order to obtain a more precise value of the constant $\xi$ and also for further astrophysical observations with the aim of getting an evaluation of the density of very low energy cosmic photons contributing to the background energy density.

\section{Conflict of Interests}

The author declares that there is no conflict of interests regarding the publication of this paper.

\section{References}

[1] B. H. Bransden and C. J. Joachain, Physics of Atoms and Molecules, Longman, New York, NY, USA, 1983.

[2] L. H. Ryder, Quantum Field Theory, Cambridge University Press, Cambridge, UK, 1987.

[3] H. Haken, Light, North Holland Publishing, Amsterdam, The Netherlands, 1981.

[4] A. I. Akhiezer and B. V. Berestetskii, Quantum Electrodynamics, Interscience Publishers, New York, NY, USA, 1965.

[5] P. W. Milonni, The Quantum Vacuum, Academic Press, Boston, Mass, USA, 1994.

[6] C. P. Enz, "Is the zero-point energy real?" in Physical Reality and Mathematical Description, C. P. Enz and J. Mehra, Eds., Reidel, Dordrecht, The Netherlands, 1974.

[7] R. Feynman, The Strange Theory of Light and Matter, Princeton University Press, Princeton, NJ, USA, 1988.

[8] S. Weinberg, “The cosmological constant problem," Reviews of Modern Physics, vol. 61, no. 1, pp. 1-23, 1989.

[9] T. Hey, The New Quantum Universe, Cambridge University Press, Cambridge, UK, 2003.

[10] S. M. Carroll, M. Hoffman, and M. Trodden, "Can the dark energy equation-of-state parameter w be less than -1?" Physical Review D, vol. 68, no. 2, Article ID 023509, 2003.

[11] V. Fanaoni, "Cumulative author index B701-B703," Nuclear Physics B, vol. 703, no. 3, pp. 557-558, 2004.

[12] S. Nojiri and S. D. Odintsov, "Gravity assisted dark energy dominance and cosmic acceleration," Physics Letters B: Nuclear, Elementary Particle and High-Energy Physics, vol. 599, no. 3-4, pp. 137-142, 2004.
[13] M. C. B. Abdalla, S. Nojiri, and S. D. Odintsov, "Consistent modified gravity: dark energy, acceleration and the absence of cosmic doomsday," Classical and Quantum Gravity, vol. 22, no. 5, pp. L35-L42, 2005.

[14] E. Elizalde, S. Nojiri, S. Odintsov, and P. Wang, "Dark energy: vacuum fluctuations, the effective phantom phase, and holography," Physical Review D, vol. 71, no. 10, Article ID 103504, 2005.

[15] J. A. Frieman, M. S. Turner, and D. Huterer, "Dark energy and the accelerating universe," Annual Review of Astronomy and Astrophysics, vol. 46, pp. 385-432, 2008.

[16] A. Silvestri and M. Trodden, "Approaches to understanding cosmic acceleration," Reports on Progress in Physics, vol. 72, no. 9, Article ID 096901, 2009.

[17] L. Labun and J. Rafelski, "Vacuum structure and dark energy," International Journal of Modern Physics D, vol. 19, no. 14, pp. 2299-2304, 2010.

[18] P. J. E. Peebles and B. Ratra, "The cosmological constant and dark energy," Reviews of Modern Physics, vol. 75, no. 2, pp. 559606, 2003.

[19] T. Padmanabhan, "Cosmological constant-the weight of the vacuum," Physics Reports, vol. 380, no. 5-6, pp. 235-320, 2003.

[20] C. Meis, "Photon wave-particle duality and virtual electromagnetic waves," Foundations of Physics, vol. 27, no. 6, pp. 865-873, 1997.

[21] M. Weissbluth, Photon-Atom Interactions, Academic Press, 1988.

[22] P. W. Milonni, "Casimir forces without the vacuum radiation field," Physical Review A, vol. 25, no. 3, pp. 1315-1327, 1982.

[23] P. Grangier, G. Roger, and A. Aspect, "Experimental evidence for a photon anticorrelation effect on a beam splitter: a new light on single-photon interferences," Europhysics Letters, vol. 1, no. 4, p. 173, 1986.

[24] J. C. Garrison and R. Y. . Chiao, Quantum Optics, Oxford University Press, Oxford, UK, 2008.

[25] F. H. Read, Electromagnetic Radiation, John Wiley \& Sons, New York, NY, USA, 1980.

[26] C. Meis, "Electric potential of the quantum vacuum," Physics Essays, vol. 22, no. 1, pp. 17-18, 2009.

[27] H. A. Bethe, "The electromagnetic shift of energy levels," Physical Review, vol. 72, no. 4, pp. 339-341, 1947.

[28] T. A. Welton, "Some observable effects of the quantum-mechanical fluctuations of the electromagnetic field," Physical Review, vol. 74, no. 9, pp. 1157-1167, 1948.

[29] R. P. Feynman, The Quantum Theory of Field, Wiley Interscience, New York, NY, USA, 1961. 

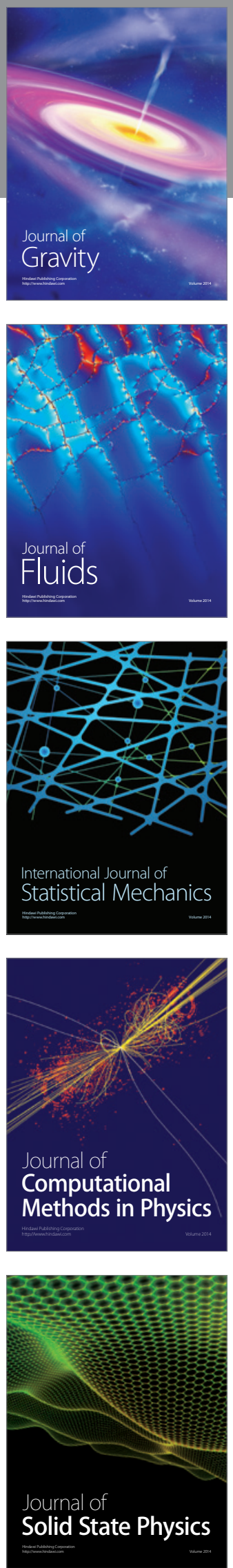

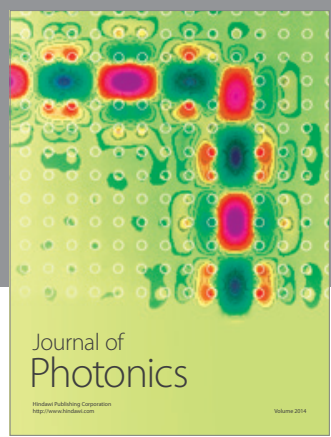

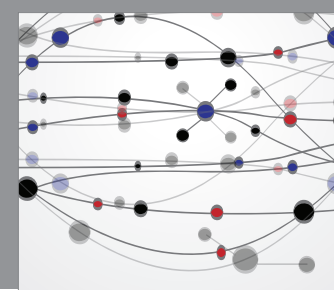

The Scientific World Journal

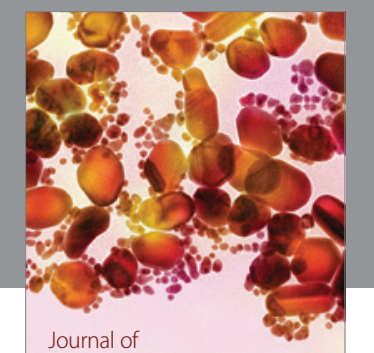

Soft Matter
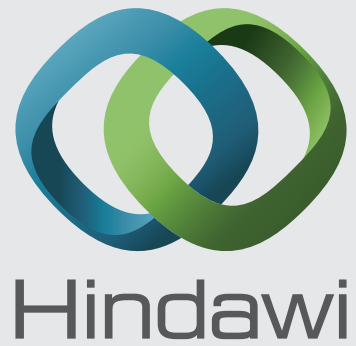

Submit your manuscripts at

http://www.hindawi.com
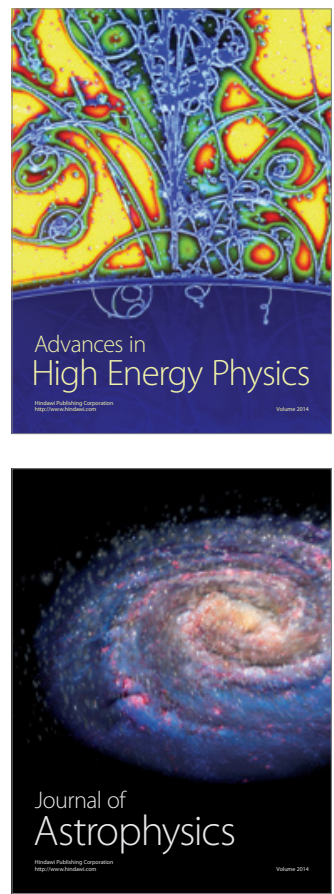
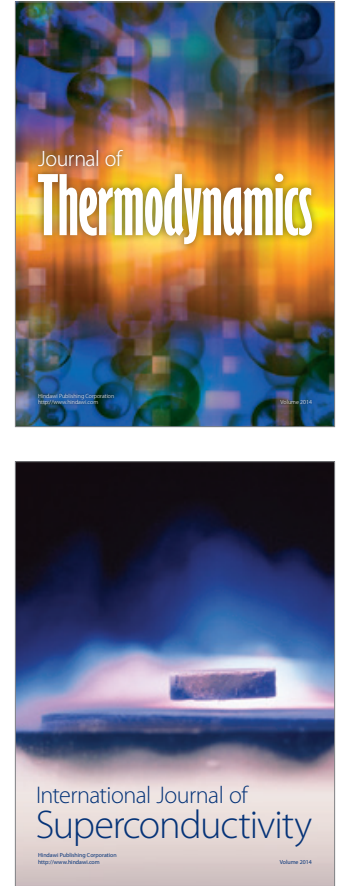
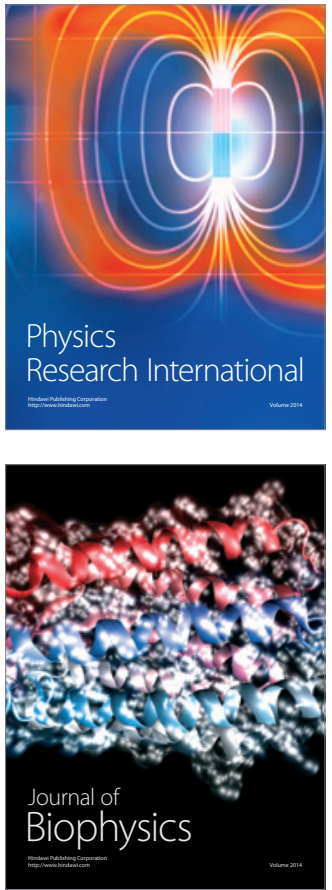
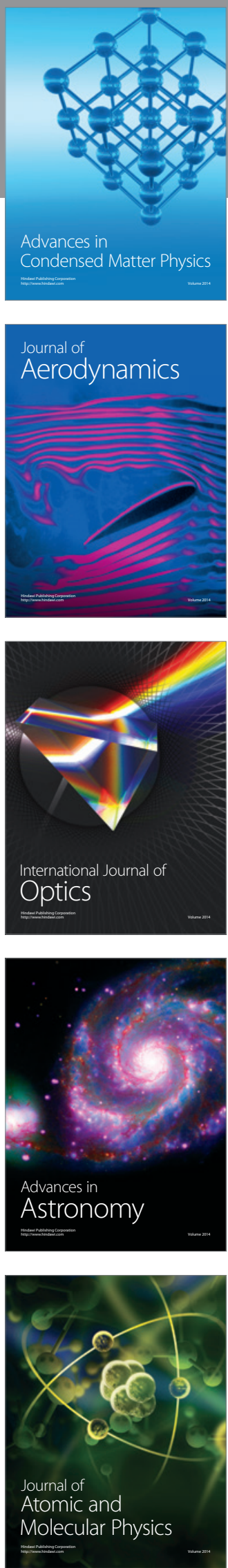